\title{
Programación lineal en la asignación de planeación de mano de obra de entidades bancarias
}

Freddy Lenin Villarreal Satama. leninv@uhemisferios.edu.ec

https://orcid.org/0000-0001-7883-1718

Facultad de Ciencias Económicas y Empresariales, Dirección de Investigación, Universidad Hemisferios (Quito-Ecuador).

\section{Heimy Paola Márquez Fuentes. heimy.marquez@ute.edu.ec Universidad Técnica Equinoccial, (Quito-Ecuador).}

Janneth Efigenia Núñez Ribadeneira jenunezr@profesores.uhemisferios.edu.ec https://orcid.org/0000-0002-3938-3542 Facultad de Ciencias Económicas y Empresariales, Universidad Hemisferios, (Quito-Ecuador)

Santiago Andrés Ullauri Betancourt. santiagou@uhemisferios.edu.ec https://orcid.org/0000-0003-0858-3178 Dirección de Investigación, Universidad Hemisferios (Quito-Ecuador).

\author{
Diego Ignacio Montenegro Gálvez \\ diegom@uhemisferios.edu.ec \\ https://orcid.org/0000-0002-9760-1181 \\ IDE Business School, Universidad Hemisferios \\ (Quito-Ecuador) \\ Daniel Fernando López Jiménez \\ daniell@uhemisferios.edu.ec \\ https://orcid.org/0000-0002-9163-8004 \\ Vicerrectorado Académico, Universidad Hemisferios \\ (Quito-Ecuador)
}




\section{RESUMEN}

El presente trabajo se basa en una propuesta de generación de un modelo de programación lineal de asignación de horarios para una entidad bancaria con la metodología simplex, cuyo objetivo es generar la optimización de recursos, minimizando el número de empleados contratados y de esta forma reducir el costo de la nómina. El modelo matemático proporciona el número mínimo requerido de personal para la sucursal con la función de minimización para el personal de caja de tiempo completo cuyo modelo puede ser emulado para situaciones similares pertinentes a la gestión de servicio en supermercados, cooperativas, mutualistas, servicios de comida rápida y atención a clientes de entidades públicas. El principal resultado de este trabajo es la asignación óptima del recurso en 16 empleados. EL modelo puede ser sensibilizado en función de las necesidades de personal en la semana, crecimiento de las entidades donde el papel de la tecnología juega un rol importante en la automatización de los servicios.

Palabras clave: programación lineal; planeación mano de obra; método simplex; minimizar costo. 


\title{
Linear programming in the allocation of workforce planning of banking entities
}

\begin{abstract}
This work is based on a proposal for the generation of a linear scheduling model for a banking entity with the simplex methodology, whose objective is to generate the optimization of resources, minimizing the number of employees hired and thus reducing the payroll cost. The mathematical model provides the minimum required number of staff for the branch with the minimization function for full-time cashier staff whose model can be emulated for similar situations pertinent to service management in supermarkets, cooperatives, mutuals, food services fast and customer service of public entities. The main result of this work is the optimal allocation of the resource in 16 employees. The model can be sensitized depending on the needs of personnel during the week, growth of the entities where the role of technology plays an important role in the automation of services.
\end{abstract}

Keywords: linear programming; workforce planning; simplex method; minimize cost.

Artículo recibido: 05 octubre. 2021 Aceptado para publicación: 02 noviembre 2021 Correspondencia: santiagou@uhemisferios.edu.ec Conflictos de Interés: Ninguna que declarar 


\section{INTRODUCCIÓN}

La programación lineal en sus primeras actividades de investigación nace en las operaciones militares requeridas de asignación de recursos y aplicaciones en tiempo real de entrega de equipo logístico en la Segunda Guerra Mundial, con la colaboración de expertos científicos en la elaboración, desarrollo y solución a problemas, que con el pasar de los años se fueron adaptando a la industria, en la medida que su aplicación genere reducción de costos en las empresas, las mismas que van en función de las estrategias y tácticas para la mejora de la productividad y eficiencia de las operaciones (Taha, Investigación de operaciones, 2011).

En la actualidad los modelos de programación matemática van de la mano con el avance de la tecnología, en el uso de software especializado para generar gran cantidad de datos y variables de simulación, cuyas soluciones se las obtiene de manera ágil y a bajo costo.

Los modelos de programación matemática ayudan a las empresas a insertarse en un proceso de mejora constante en las operaciones, plantas de producción, horarios de asignación flexibles, cuyo impacto es directamente proporcional a los niveles de productividad deseados y la obtención de eficiencia técnica propuesta por Farrell, como una medida satisfactoria de eficiencia productiva, que toma en cuenta todos los inputs o recursos empleados generado una salida llamada output que es el resultado de la eficiencia (Coll \& Olga, 2006).

Como rama de las matemáticas, el campo de aplicación es diverso como en la selección de portafolios de inversión y asignación óptima de las carteras de crédito, programación de mano de obra, programación de la producción, mezcla de productos, selección de medios de comunicación, problemas de dietas en centros hospitalarios y veterinaria, problemas de mezclas de ingredientes, problemas de transporte y embarques, entre otros.

En los problemas de programación de mano de obra, hace referencia a las necesidades de personal de tiempo completo, medio tiempo y tiempo parcial en períodos prolongados de trabajo o trabajos de temporada, en los que las entidades hacen uso de la programación lineal para la asignación de turnos de trabajo en la atención al cliente, tomando en cuenta las horas de mayor afluencia de usuarios en el balcón de servicios, cajas, de manera que el servicio no se vea afectado por reclamos constantes de clientes que solicitan una atención ágil. Las aplicaciones de la programación lineal se muestran a continuación:

Figura 1. Aplicaciones a la programación lineal 


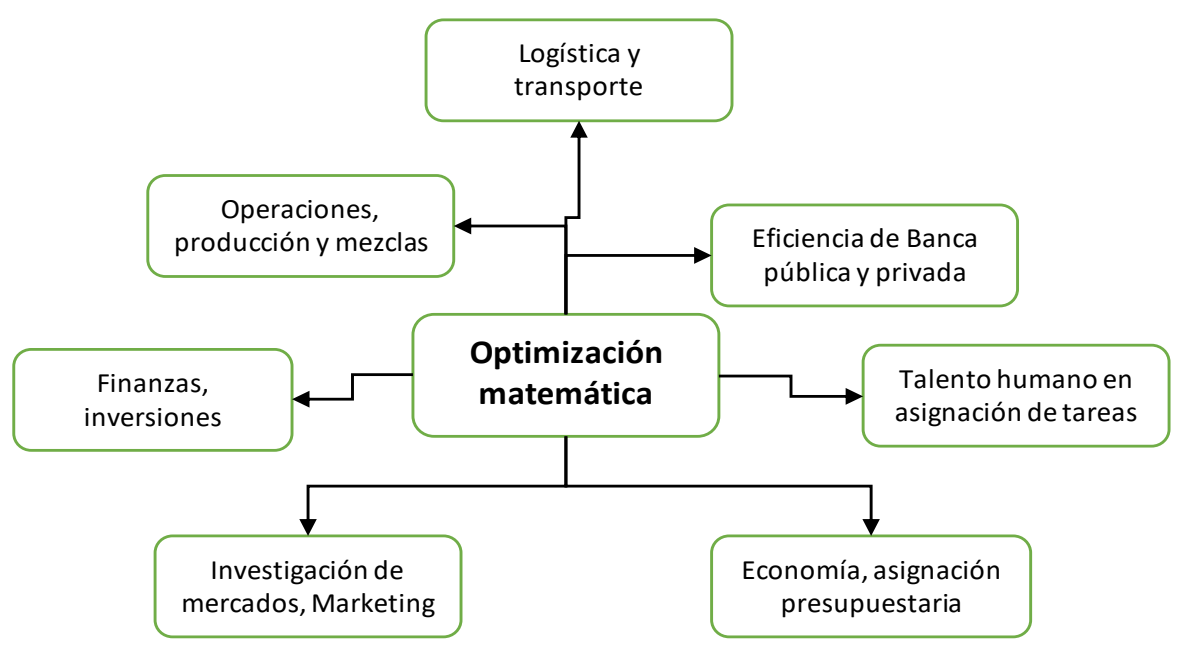

Fuente: Elaboración propia, tomada de (Render, Stair, \& Hanna, 2012)

La programación lineal constituye una herramienta valiosa en la solución a problemas como se aprecia en la figura 1, que involucra la adaptación de algoritmos para resolver problemas específico s particulares, por lo tanto se convierte en una ciencia y arte por el hecho de que el arte está en el diseño de prototipos de prueba previo a su aplicación final, el uso de la investigación científica, y es una ciencia ya que hace uso de la técnica matemática basada en el álgebra matricial y a su vez depende de la inspiración del investigador para tratar de maximizar los beneficios o minimizar el uso de recursos como es el de este trabajo en la minimización de tiempo y costos de las asignaciones de turnos de trabajo, para dar una respuesta efectiva en las necesidades de talento humano que requieren los directivos en las diferentes entidades públicas o privadas.

\section{REVISIÓN BIBLIOGRÁFICA}

(Acuña, Madiedo, \& Ortíz, 2013) genera en su trabajo un modelo matemático para la asignación de proyectos en carga de trabajo, basada en una prueba de hipótesis de Bernoulli, que minimiza la diferencia entre la carga máxima y mínima de trabajo, y en los dos modelos utilizados los resultados no coinciden en un $90 \%$.

(Ojeda, 2015) en su trabajo de asignación de horarios al personal en una empresa telefónica de México mediante un algoritmo genético donde se obtiene como resultado principal que cada turno cubra un horario pertinente de 28 intervalos de cuarto de hora en horario diurno y en horario nocturno cubre 24 intervalos de cuarto de hora, encontrándose evidencia cuantitativa que se ajusta a la curva de demanda.

La asignación de turnos mediante metaheurísticas han sido estudiadas en cuatro empresas asturianas por (De la Fuente, Lozano, \& Isabel, 1998), donde cada empresa tiene necesidades particulares para disminuir el número de horas de semanales de trabajo con una nueva metodología de asignación de 
turnos basada en la programación entera y la técnica heurística como el tabú search que proporciona una solución aceptable cuyos resultados respetan el requisito de ciclicidad y el equilibrio de turnos para los trabajadores.

La programación de turnos eficientes en las actividades de enfermería es crucial para la calidad del servicio en la que se evidencia el gran trabajo de cerrar brechas entre la práctica y los modelos académicos para que tengan una mejor utilidad en el trabajo real con la capacidad de generar modelos complejos. Su enfoque es la revisión literaria, en las que destacan los modelos heurísticos en algoritmos genéticos y técnicas de diversificación e intensificación como los óptimos locales, rompiendo el uso de los modelos lineales tradicionales y finalmente expone que los modelos mayormente aplicados para casos de asignación de enfermería utilizan la técnica de programación lineal entera $(40,74 \%)$, de la literatura revisada y modelos multiobjetivo con $(31,48 \%)$ (Arias, Bautista, \& Meneses, 2019).

Bermúdez (2011) genera un estudio documental informativo, en el que aborda los aportes de la comunidad científica, respecto a temas relacionados con programación lineal, en las que evidencia que los modelos de optimización basados en programación lineal son utilizados en ambientes reales para la solución de problemas y ayuda a la toma de decisiones que contribuyen a la reducción de costos, mejorar la productividad en la planificación de la producción para el aprovechamiento de la capacidad de reducción en inventarios.

Por otro lado (Cavada, Cortés, \& Rey, 2012), en su trabajo de un modelo de asignación de personal para un aeropuerto, cuyo objetivo es determinar el personal necesario para operar el patio de equipajes mediante la metodología de programación lineal entera, con una base de datos histórica y luego con una base de demanda generada de asignación de operarios con un modelo de partición de conjuntos, validado por una empresa de operación de equipajes de un aeropuerto para lograr mejoras productivas.

En el caso de manejo y gestión de proyectos, la investigación de operaciones es útil como lo señala Terrazas (2012) para la mejor asignación de recursos en actividades competidoras. En el análisis de factibilidad de proyectos una dificultad es saber ubicar un proyecto en las mejores condiciones operativas para minimizar costos y maximizar ganancias.

(León, Díaz, \& Contreras, 2007), describen en su trabajo un modelo de programación lineal para la asignación de turnos a máquinas de una empresa manufacturera, considerando prioridades basadas en la planificación de la planta donde la característica no se sustenta en la velocidad de las máquinas, puesto que ello no es significativo, sino en las características técnicas para un trabajo de calidad y 
eficiente en la entrega de productos basados en el plan maestro de producción MPR II, mejorando todos los procesos de la cadena de valor.

Las compañías de retail, realizan la programación de visitas con el diseño de jornadas de horario semanal y secuenciamiento de visitas adaptada a la demanda de clientes basado en la técnica de la programación lineal entera mixta de las compañías SC Johnson, cuyo resultado principal de este enfoque cubren una mayor área de ventas de $68 \%$ a $88 \%$ con el uso de los mismos recursos de personal (Miranda, 2017).

Un importante estudio es el realizado por (Pérez, Navarro, \& Rocha, 2019), en el que exponen su trabajo de asignación de clientes hacia asesores comerciales en una empresa de tecnología para satisfacer la demanda de perfiles apropiados y con ello generar un plan de contratación de nuevos asesores que en total serían 29 de los 35 iniciales que se encontraba planificado, que hablen varios idiomas, para cubrir la demanda insatisfecha del 99,20\% y sus expectativas de la organización, basado en la técnica de la programación lineal entera mixta.

En las plantaciones forestales es importante la asignación de turnos técnicos con el criterio de máxima productividad y máximo valor esperado de la tierra basado en la técnica de programación lineal entera en la determinación de turnos mono criterios, y también utilizando la técnica de programación por metas ponderada y lexicográfica entera en la determinación de turnos múltiples objetivos que variaron entre 31 y 35 como turnos técnicamente óptimos. (Mariel, Aleixo, \& Alvares, 2019).

\section{METODOLOGÍA}

\section{El modelo de programación lineal}

Para Taha (2012) la solución al modelo matemático responde a si satisface todas las restricciones y a su vez se optimiza cuando genera el mejor valor posible. La programación lineal parte de la declaración de la función objetivo y las restricciones inherentes a los recursos disponibles y que a su vez son escasos. Existen otros métodos disponibles para la solución de problemas como es el método de programación entera, programación dinámica, programación en red, programación no lineal.

Se precisa el algoritmo matemático que obedece a las variables, que nos define el dominio en el que se encuentra la solución óptima, y las restricciones lineales como un conjunto de ecuaciones e inecuaciones que muestran las limitaciones empresariales de recursos, sin embargo, no todos los problemas de optimización matemática pueden ser resueltos con algoritmos, por lo que es necesario acudir a la solución heurística y metaheurística. 


\section{Fases del estudio de programación matemática}

Este tipo de modelos, tal como ocurre en proyectos tecnológicos de desarrollo de aplicaciones, debe trabajarse en sociedad, donde los expertos en la modelación matemática trabajan en conjunto con quiénes demandan la solución a un problema; considerándose así a la programación matemática como una ciencia y un arte. El arte es el modelado y la habilidad para desarrollar el modelo, y la ciencia está en la matemática con juicio técnico. Soot (2018) afirma que sin importar el campo del cual provenga el problema y lo específico del tema abordado, se analiza el proceso con un modelo matemático que contiene las fases de la programación matemática, tal como se indica en la siguiente figura:

Figura 2. Proceso de modelado de programación matemática

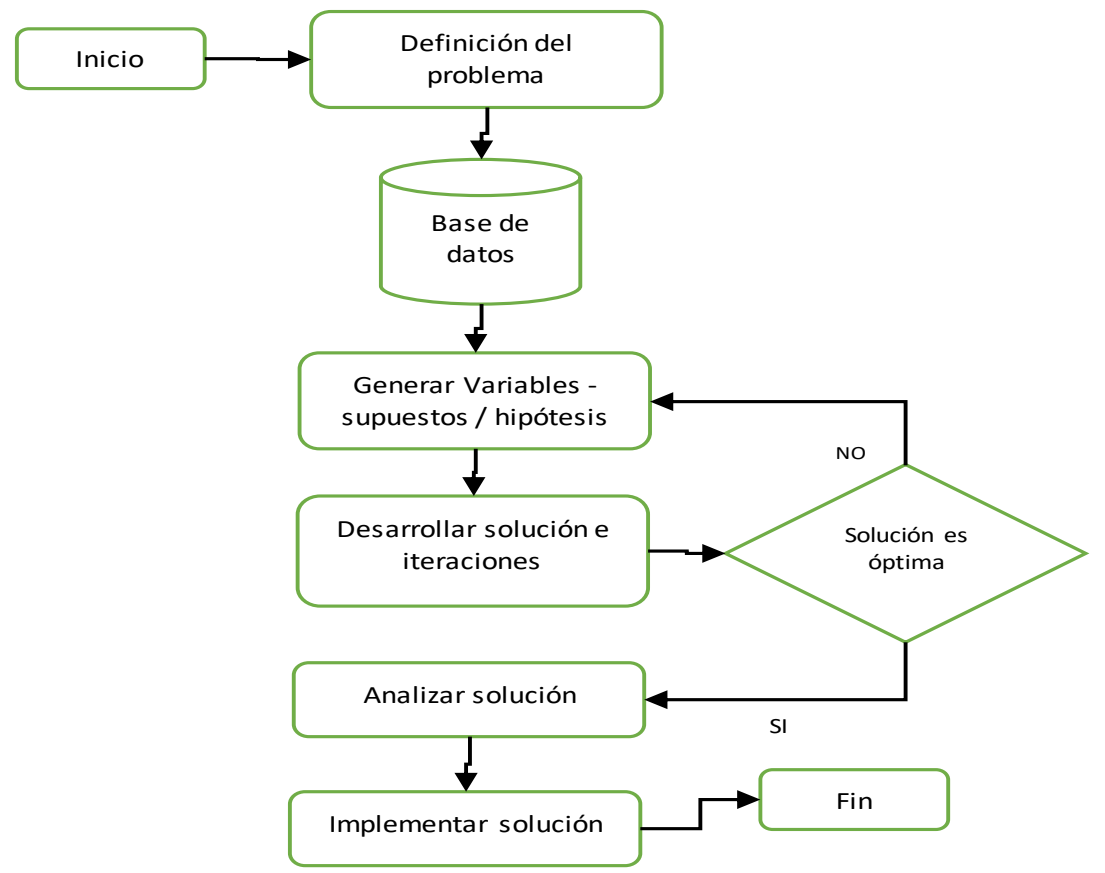

Fuente: Realización propia basado en (Render, Stair, \& Hanna, 2012)

\section{El método Simplex (Izar, 1996)}

Es un procedimiento general de programación matemática para llegar a la solución óptima que se obtiene en un número finito de pasos. Estos son:

- La solución es cualquier conjunto de variables $X_{j}$ que satisfacen las restricciones del problema $(A x=B)$.

- La solución factible es aquella que satisface la no - negatividad de las restricciones $\left(X_{j \geq 0}\right)$.

- La solución básica del sistema de $m$ ecuaciones lineales son $n$ variables $A x=B,(m<n)$, cuyo rango $R(A)=m$ (variables básicas). La solución es $n-m=0 ; \mathrm{y}$, resolviendo para las $m$ variables restantes, cuando el determinante de sus coeficientes no sean cero. 
- La solución básica factible es tal, que todas las $m$ variables básicas $x_{j} \geq 0$.

- La degeneración de una solución básica $(A x=B)$ se da si una o más variables básicas son iguales a cero.

Figura 3. Proceso de metodología Simplex

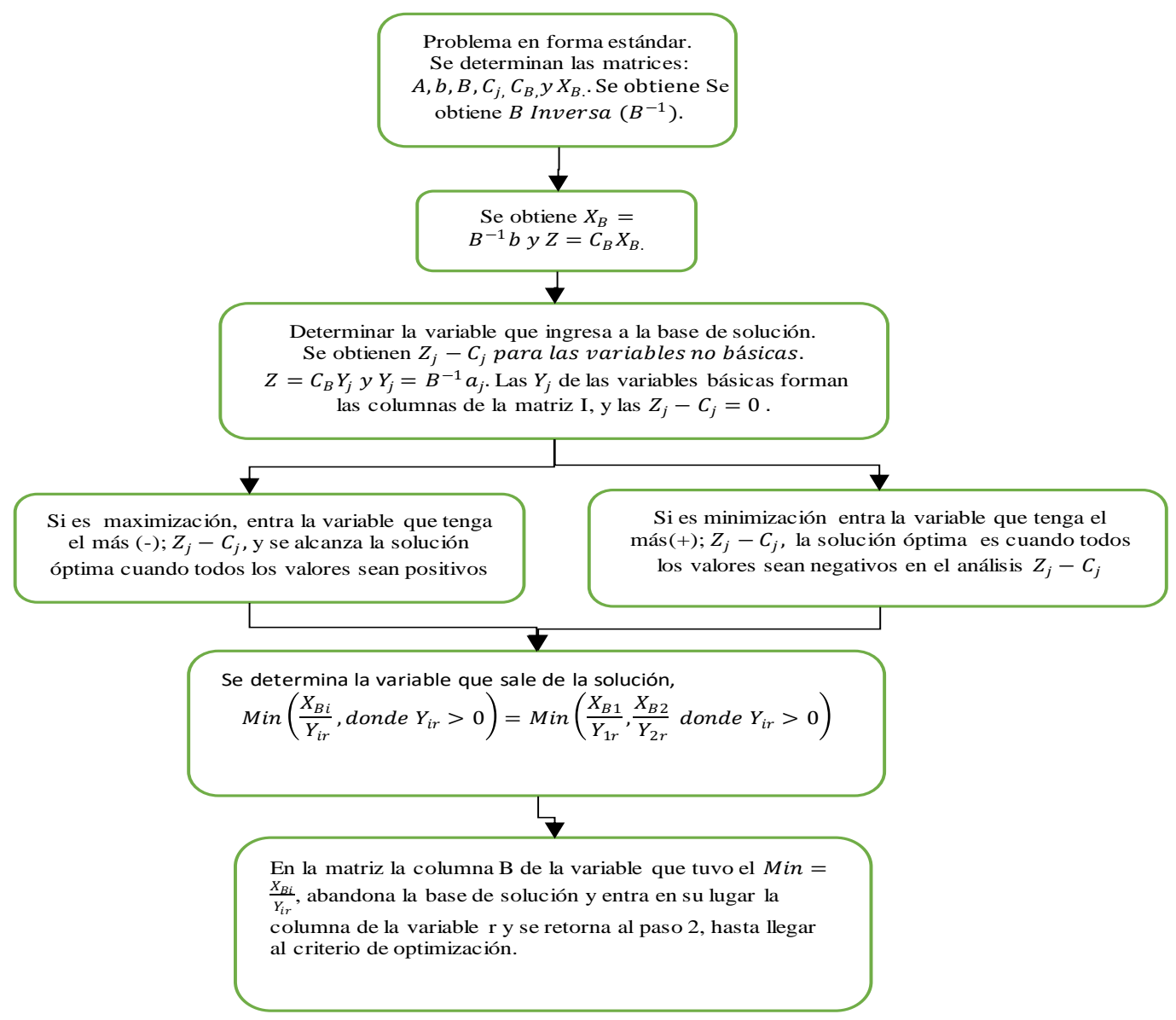

Fuente: Notas del método simplex. Martínez, H.

Tabla1. Matriz Método Simplex.

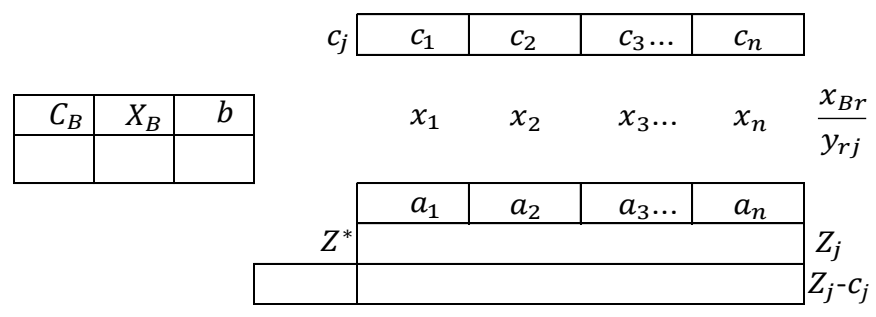

Fuente: Notas del método simplex. Martínez, $H$. 


\section{Donde:}

$X_{B}=$ Vector de la solución básica factible.

$b=$ variables básicas.

$Z^{*}=$ función objetivo (Max - Min)

$C_{j}=$ Vector de costos y $C_{B}=$ Vector de componentes $\mathrm{C}$

$Z_{j}=C_{B} X_{B} \quad y \quad Z_{j}-C_{j}=C_{B} X_{B}-C_{j}$

$y_{r j}=$ Nueva solución básica factible

\section{Método de las dos fases}

Este método elimina el uso de la constante $M$, siendo la fase I el proceso por el cual se trata de encontrar la solución básica inicial, y si esta aparece, se invoca ala fase II que dará la solución al problema original donde las variables deben ser reales y mayores a cero, el detalle de estas fases se describe a continuación (Mathstools, 2021):

- Fase I, Añadir $k$ columnas mediante variables artificiales a la matriz $A$, de modo que contenga una matriz identidad de tamaño $m \times n$.

- Cambiar la función objetivo original por ceros a excepción de la última $k$ componentes.

- Generar el algoritmo que puede dar lugar a la solución óptima, en el que las variables artificiales sales de la base para dar paso a la fase II, o puede darse el caso de que el problema arroje una solución óptima finita diferente de cero, por lo que el problema original no tendría solución.

- Fase II, eliminadas las variables artificiales usando la función objetivo original.

- Tomar las m primeras columnas de la matriz $A$.

- Seguir el algoritmo hasta llegar a las cuatro posibles salidas del problema.

\section{DATOS, DEFINICIÓN DE VARIABLES Y MODELO MATEMÁTICO}

En la tabla siguiente, se recogen los datos e información del banco, en referencia al período de atención. El número de personal de caja se encuentra en función de la frecuencia de clientes que se acercan a la agencia para realizar sus transacciones y el horario de trabajo de ocho horas diarias al día, tomando en cuenta el número de personas requeridas por cada día de la semana. 
Tabla 2. Datos de requerimiento de personal y asignación diaria.

\begin{tabular}{lcccccccc}
\multicolumn{1}{c}{ Día } & No personas & \multicolumn{7}{c}{ Tabla de planificación } \\
\hline Lunes & 10 & $\mathrm{X} 1$ & $\mathrm{D}$ & $\mathrm{D}$ & $\mathrm{X} 4$ & $\mathrm{X} 5$ & $\mathrm{X} 6$ & $\mathrm{X} 7$ \\
\hline Martes & 9 & $\mathrm{X} 1$ & $\mathrm{X} 2$ & $\mathrm{D}$ & $\mathrm{D}$ & $\mathrm{X} 5$ & $\mathrm{X} 6$ & $\mathrm{X} 7$ \\
\hline Miércoles & 8 & $\mathrm{X} 1$ & $\mathrm{X} 2$ & $\mathrm{X} 3$ & $\mathrm{D}$ & $\mathrm{D}$ & $\mathrm{X} 6$ & $\mathrm{X} 7$ \\
\hline Jueves & 11 & $\mathrm{X} 1$ & $\mathrm{X} 2$ & $\mathrm{X} 3$ & $\mathrm{X} 4$ & $\mathrm{D}$ & $\mathrm{D}$ & $\mathrm{X} 7$ \\
\hline Viernes & 13 & $\mathrm{X} 1$ & $\mathrm{X} 2$ & $\mathrm{X} 3$ & $\mathrm{X} 4$ & $\mathrm{X} 5$ & $\mathrm{D}$ & $\mathrm{D}$ \\
\hline Sábado & 15 & $\mathrm{D}$ & $\mathrm{X} 2$ & $\mathrm{X} 4$ & $\mathrm{X} 4$ & $\mathrm{X} 5$ & $\mathrm{X} 6$ & $\mathrm{D}$ \\
\hline Domingo & 12 & $\mathrm{D}$ & $\mathrm{D}$ & $\mathrm{X} 3$ & $\mathrm{X} 4$ & $\mathrm{X} 5$ & $\mathrm{X} 6$ & $\mathrm{X} 7$ \\
\hline
\end{tabular}

Fuente: Recolección de datos de agencia bancaria en Quito (2021).

Como se puede apreciar el lunes se requieren diez personas para atención al público, martes nueve seguido de ocho el miércoles y respectivamente hasta llegar al domingo donde hay una mayor afluencia de clientes en el que se requieren doce personas de atención. En la parte correspondiente a la tabla de planificación se puede aprecia que la variable X1 se encuentra asignada de lunes a viernes lo que responde a los cinco días laborables de esa persona con dos días de descanso (D), de la misma manera la variable X2 que corresponde a la persona que trabaja del día martes a sábado con dos días de descanso que en este caso sería domingo y lunes, siguiendo su correspondencia al trabajador X7 que ingresa a trabajar el día domingo y se cumple los cinco días laborables hasta el jueves de la próxima semana con descanso de viernes y sábado que completaría su jornada laboral. Este tipo de escenario es importante indicar que varía en función del tamaño de cada agencia bancaria, al mismo tiempo que su aplicación se deriva para los horarios asignados en supermercados, centros comerciales, cooperativas y entidades que dispongan de servicios similares, tomando en cuenta que las unidades económicas deben decidir el número de personas de caja que deben atender en la franja horaria y días laborales asignados de trabajo, por lo que las variables se definen de esta forma:

\section{Definición de variables}

$x 1$ = número de personas de contrato fijo que trabajan el lunes

$x 2=$ número de personas de contrato fijo que trabajan el martes

$x 3$ = número de personas de contrato fijo que trabajan el miércoles

$x 4=$ número de personas de contrato fijo que trabajan el jueves 
$x 5=$ número de personas de contrato fijo que trabajan el viernes

$x 6$ = número de personas de contrato fijo que trabajan el sábado

$x 7=$ número de personas de contrato fijo que trabajan el domingo

\section{Modelo de programación matemática}

Generadas las variables, se escriben las expresiones matemáticas para la función objetivo y las restricciones identificadas en la definición de las variables. Las restricciones de no negatividad se expresan de manera intuitiva en el modelo las mismas que son iguales o mayores que cero.

Función objetivo: minimizar el el número de personas en cajas.

$$
\operatorname{Min} Z=x 1+x 2+x 3+x 4+x 5+x 6+x 7
$$

\section{Sujeto a las siguientes restricciones:}

$$
\begin{aligned}
& 1 \mathrm{X} 1+0 \mathrm{X} 2+0 \mathrm{X} 3+1 \mathrm{X} 4+1 \mathrm{X} 5+1 \mathrm{X} 6+1 \mathrm{X} 7 \geq 10 \\
& 1 \mathrm{X} 1+1 \mathrm{X} 2+0 \mathrm{X} 3+0 \mathrm{X} 4+1 \mathrm{X} 5+1 \mathrm{X} 6+1 \mathrm{X} 7 \geq 9 \\
& 1 \mathrm{X} 1+1 \mathrm{X} 2+1 \mathrm{X} 3+0 \mathrm{X} 4+0 \mathrm{X} 5+1 \mathrm{X} 6+1 \mathrm{X} 7 \geq 8 \\
& 1 \mathrm{X} 1+1 \mathrm{X} 2+1 \mathrm{X} 3+1 \mathrm{X} 4+0 \mathrm{X} 5+0 \mathrm{X} 6+1 \mathrm{X} 7 \geq 11 \\
& 1 \mathrm{X} 1+1 \mathrm{X} 2+1 \mathrm{X} 3+1 \mathrm{X} 4+1 \mathrm{X} 5+0 \mathrm{X} 6+0 \mathrm{X} 7 \geq 13 \\
& 0 \mathrm{X} 1+1 \mathrm{X} 2+1 \mathrm{X} 3+1 \mathrm{X} 4+1 \mathrm{X} 5+1 \mathrm{X} 6+0 \mathrm{X} 7 \geq 15 \\
& 0 \mathrm{X} 1+0 \mathrm{X} 2+1 \mathrm{X} 3+1 \mathrm{X} 4+1 \mathrm{X} 5+1 \mathrm{X} 6+1 \mathrm{X} 7 \geq 14 \\
& \quad x 1+x 2+x 3+x 4+x 5+x 6+x 7 \geq 0(\text { No negatividad) }
\end{aligned}
$$

\section{Modelo matemático de programación con la forma estándar:}

Pasamos el problema a la forma estándar, añadiendo variables de exceso, holgura, y artificiales según corresponda (mostrar/ocultar detalles)

- Como la restricción 1 es del tipo ' $\geq '$ se agrega la variable de exceso X8 y la variable artificial $\mathrm{X} 15$.

- Como la restricción 2 es del tipo ' $\geq$ ' se agrega la variable de exceso X9 y la variable artificial $\mathrm{X} 16$.

- Como la restricción 3 es del tipo ' $\geq$ ' se agrega la variable de exceso X10 y la variable artificial $\mathrm{X} 17$.

- Como la restricción 4 es del tipo ' $\geq$ ' se agrega la variable de exceso X11 y la variable artificial $\mathrm{X} 18$.

- Como la restricción 5 es del tipo ' $\geq$ ' se agrega la variable de exceso X12 y la variable artificial X19. 
- Como la restricción 6 es del tipo ' $\geq$ ' se agrega la variable de exceso X13 y la variable artificial $\mathrm{X} 20$.

- Como la restricción 7 es del tipo ' $\geq$ ' se agrega la variable de exceso X14 y la variable artificial $\mathrm{X} 21$.

FO: MINIMIZAR: $\mathrm{Z}=\mathrm{X} 1+\mathrm{X} 2+\mathrm{X} 3+\mathrm{X} 4+\mathrm{X} 5+\mathrm{X} 6+\mathrm{X} 7$

sujeto a

$$
\begin{aligned}
& 1 X 1+0 X 2+0 X 3+1 X 4+1 X 5+1 X 6+1 X 7 \geq 10 \\
& 1 X 1+1 X 2+0 X 3+0 X 4+1 X 5+1 X 6+1 X 7 \geq 9 \\
& 1 X 1+1 X 2+1 X 3+0 X 4+0 X 5+1 X 6+1 X 7 \geq 8 \\
& 1 X 1+1 X 2+1 X 3+1 X 4+0 X 5+0 X 6+1 X 7 \geq 11 \\
& 1 X 1+1 X 2+1 X 3+1 X 4+1 X 5+0 X 6+0 X 7 \geq 13 \\
& 0 X 1+1 X 2+1 X 3+1 X 4+1 X 5+1 X 6+0 X 7 \geq 15 \\
& 0 X 1+0 X 2+1 X 3+1 X 4+1 X 5+1 X 6+1 X 7 \geq 14
\end{aligned}
$$

$$
\mathrm{X} 1, \mathrm{X} 2, \mathrm{X} 3, \mathrm{X} 4, \mathrm{X} 5, \mathrm{X} 6, \mathrm{X} 7 \geq 0
$$

Pasamos a construir la primera tabla de la Fase I del método de las Dos Fases.

MAXIMIZAR: $Z=-1 X 1-1 \mathrm{X} 2-1 \mathrm{X} 3-1 \mathrm{X} 4-1 \mathrm{X} 5-1 \mathrm{X} 6-1 \mathrm{X} 7+0 \mathrm{X} 8+0 \mathrm{X} 9+0 \mathrm{X} 10+0 \mathrm{X} 11$ $+0 \mathrm{X} 12+0 \mathrm{X} 13+0 \mathrm{X} 14+0 \mathrm{X} 15+0 \mathrm{X} 16+0 \mathrm{X} 17+0 \mathrm{X} 18+0 \mathrm{X} 19+0 \mathrm{X} 20+0 \mathrm{X} 21$

sujeto a

$$
\begin{aligned}
& 1 \mathrm{X} 1+1 \mathrm{X} 4+1 \mathrm{X} 5+1 \mathrm{X} 6+1 \mathrm{X} 7-1 \mathrm{X} 8+1 \mathrm{X} 15=10 \\
& 1 \mathrm{X} 1+1 \mathrm{X} 2+1 \mathrm{X} 5+1 \mathrm{X} 6+1 \mathrm{X} 7-1 \mathrm{X} 9+1 \mathrm{X} 16=9 \\
& 1 \mathrm{X} 1+1 \mathrm{X} 2+1 \mathrm{X} 3+1 \mathrm{X} 6+1 \mathrm{X} 7-1 \mathrm{X} 10+1 \mathrm{X} 17=8 \\
& 1 \mathrm{X} 1+1 \mathrm{X} 2+1 \mathrm{X} 3+1 \mathrm{X} 4+1 \mathrm{X} 7-1 \mathrm{X} 11+1 \mathrm{X} 18=11 \\
& 1 \mathrm{X} 1+1 \mathrm{X} 2+1 \mathrm{X} 3+1 \mathrm{X} 4+1 \mathrm{X} 5-1 \mathrm{X} 12+1 \mathrm{X} 19=13 \\
& 0 \mathrm{X} 1+1 \mathrm{X} 2+1 \mathrm{X} 3+1 \mathrm{X} 4+1 \mathrm{X} 5+1 \mathrm{X} 6-1 \mathrm{X} 13+1 \mathrm{X} 20=15 \\
& 0 \mathrm{X} 1+1 \mathrm{X} 3+1 \mathrm{X} 4+1 \mathrm{X} 5+1 \mathrm{X} 6+1 \mathrm{X} 7-1 \mathrm{X} 14+1 \mathrm{X} 21=14
\end{aligned}
$$

X1, X2, X3, X4, X5, X6, X7, X8, X9, X10, X11, X12, X13, X14, X15, X16, X17, X18, X19, $\mathrm{X} 20, \mathrm{X} 21 \geq 0$ 


\section{RESULTADOS Y DISCUSIÓN}

Para efectos de presentación, se mostrará a continuación las matrices de los resultados generados en el programa de investigación operativa PHP-Simplex. Para llegar al resultado deseado con el método simplex aplicando la técnica de las dos fases, el modelo indica que deben pasar por diez interacciones hasta llegar a la solución básica factible. Se presentan las cuatro primeras interacciones y el resto se presenta en los anexos.

El resultado de la solución básica factible indica que hay infinitos valores para las variables $x 1$ a $x 7$ y que luego de cumplir los requisitos de la primera y segunda fase, el valor de la función objetivo de minimizar el número de personas a ser contratadas es de 16. Si bien es cierto este planteamiento aplica para la agencia donde se tomaron los datos, el algoritmo para el modelo matemático establecido puede ser adaptado para el tamaño de la agencia, el número de empleados de tiempo completo, medio tiempo y tiempo parcial, horarios de atención en función de las horas donde la concurrencia de clientes es mayor. En el caso de la agencia en el que se estableció este estudio, en el horario de trabajo de tiempo completo de la franja horaria de 09:00 a 17:00 es necesario contar el lunes con un cajero, el martes con dos cajeros, el día miércoles con dos cajeros, el día jueves con cinco caeros, para el día viernes con tres cajeros, para el día sábado dos y domingo un cajero, de esta forma minimiza el número de personal requerido para la agencia bancaria. 
Tabla 3. Resultado de la primera interacción del método simplex

\begin{tabular}{ccccccccccccccccccccccccccc}
\hline Tabla 1 & & $\mathbf{0}$ & $\mathbf{0}$ & $\mathbf{0}$ & $\mathbf{0}$ & $\mathbf{0}$ & $\mathbf{0}$ & $\mathbf{0}$ & $\mathbf{0}$ & $\mathbf{0}$ & $\mathbf{0}$ & $\mathbf{0}$ & $\mathbf{0}$ & $\mathbf{0}$ & $\mathbf{0}$ & $\mathbf{- 1}$ & $\mathbf{- 1}$ & $\mathbf{- 1}$ & $\mathbf{- 1}$ & $\mathbf{- 1}$ & $\mathbf{- 1}$ & $\mathbf{- 1}$ \\
\hline Base & $\mathbf{C b}$ & $\mathbf{P 0}$ & $\mathbf{P 1}$ & $\mathbf{P 2}$ & $\mathbf{P 3}$ & $\mathbf{P 4}$ & $\mathbf{P 5}$ & $\mathbf{P 6}$ & $\mathbf{P 7}$ & $\mathbf{P 8}$ & $\mathbf{P 9}$ & $\mathbf{P 1 0}$ & $\mathbf{P 1 1}$ & $\mathbf{P 1 2}$ & $\mathbf{P 1 3}$ & $\mathbf{P 1 4}$ & $\mathbf{P 1 5}$ & $\mathbf{P 1 6}$ & $\mathbf{P 1 7}$ & $\mathbf{P 1 8}$ & $\mathbf{P 1 9}$ & $\mathbf{P 2 0}$ & $\mathbf{P 2 1}$ \\
\hline P15 & -1 & 10 & 1 & 0 & 0 & 1 & 1 & 1 & 1 & -1 & 0 & 0 & 0 & 0 & 0 & 0 & 1 & 0 & 0 & 0 & 0 & 0 & 0 \\
\hline P16 & -1 & 9 & 1 & 1 & 0 & 0 & 1 & 1 & 1 & 0 & -1 & 0 & 0 & 0 & 0 & 0 & 0 & 1 & 0 & 0 & 0 & 0 & 0 \\
\hline P17 & -1 & 8 & 1 & 1 & 1 & 0 & 0 & 1 & 1 & 0 & 0 & -1 & 0 & 0 & 0 & 0 & 0 & 0 & 1 & 0 & 0 & 0 & 0 \\
\hline P18 & -1 & 11 & 1 & 1 & 1 & 1 & 0 & 0 & 1 & 0 & 0 & 0 & -1 & 0 & 0 & 0 & 0 & 0 & 0 & 1 & 0 & 0 & 0 \\
\hline P19 & -1 & 13 & 1 & 1 & 1 & 1 & 1 & 0 & 0 & 0 & 0 & 0 & 0 & -1 & 0 & 0 & 0 & 0 & 0 & 0 & 1 & 0 & 0 \\
\hline P20 & -1 & 15 & 0 & 1 & 1 & 1 & 1 & 1 & 0 & 0 & 0 & 0 & 0 & 0 & -1 & 0 & 0 & 0 & 0 & 0 & 0 & 1 & 0 \\
\hline P21 & -1 & 14 & 0 & 0 & 1 & 1 & 1 & 1 & 1 & 0 & 0 & 0 & 0 & 0 & 0 & -1 & 0 & 0 & 0 & 0 & 0 & 0 & 1 \\
\hline $\mathbf{Z}$ & & -80 & -5 & -5 & -5 & -5 & -5 & -5 & -5 & 1 & 1 & 1 & 1 & 1 & 1 & 1 & 0 & 0 & 0 & 0 & 0 & 0 & 0 \\
\hline
\end{tabular}

Fuente: Elaboración propia, PHP-Simplex

De acuerdo con la técnica del método simplex se indica la variable que "sale" de la base es P17 y a su vez la variable que "entra" es P1 que se encuentra en la misma columna. 
Tabla 4. Resultado de la segunda interacción del método simplex

\begin{tabular}{ccccccccccccccccccccccccc}
\hline \multicolumn{2}{c}{ Tabla 2} & & $\mathbf{0}$ & $\mathbf{0}$ & $\mathbf{0}$ & $\mathbf{0}$ & $\mathbf{0}$ & $\mathbf{0}$ & $\mathbf{0}$ & $\mathbf{0}$ & $\mathbf{0}$ & $\mathbf{0}$ & $\mathbf{0}$ & $\mathbf{0}$ & $\mathbf{0}$ & $\mathbf{0}$ & $\mathbf{- 1}$ & $\mathbf{- 1}$ & $\mathbf{- 1}$ & $\mathbf{- 1}$ & $\mathbf{- 1}$ & $\mathbf{- 1}$ & $\mathbf{- 1}$ \\
\hline Base & Cb & P0 & P1 & P2 & P3 & P4 & P5 & P6 & P7 & P8 & P9 & P10 & P11 & P12 & P13 & P14 & P15 & P16 & P17 & P18 & P19 & P20 & P21 \\
\hline P15 & -1 & 2 & 0 & -1 & -1 & 1 & 1 & 0 & 0 & -1 & 0 & 1 & 0 & 0 & 0 & 0 & 1 & 0 & -1 & 0 & 0 & 0 & 0 \\
\hline P16 & -1 & 1 & 0 & 0 & -1 & 0 & 1 & 0 & 0 & 0 & -1 & 1 & 0 & 0 & 0 & 0 & 0 & 1 & -1 & 0 & 0 & 0 & 0 \\
\hline P1 & 0 & 8 & 1 & 1 & 1 & 0 & 0 & 1 & 1 & 0 & 0 & -1 & 0 & 0 & 0 & 0 & 0 & 0 & 1 & 0 & 0 & 0 & 0 \\
\hline P18 & -1 & 3 & 0 & 0 & 0 & 1 & 0 & -1 & 0 & 0 & 0 & 1 & -1 & 0 & 0 & 0 & 0 & 0 & -1 & 1 & 0 & 0 & 0 \\
\hline P19 & -1 & 5 & 0 & 0 & 0 & 1 & 1 & -1 & -1 & 0 & 0 & 1 & 0 & -1 & 0 & 0 & 0 & 0 & -1 & 0 & 1 & 0 & 0 \\
\hline P20 & -1 & 15 & 0 & 1 & 1 & 1 & 1 & 1 & 0 & 0 & 0 & 0 & 0 & 0 & -1 & 0 & 0 & 0 & 0 & 0 & 0 & 1 & 0 \\
\hline P21 & -1 & 14 & 0 & 0 & 1 & 1 & 1 & 1 & 1 & 0 & 0 & 0 & 0 & 0 & 0 & -1 & 0 & 0 & 0 & 0 & 0 & 0 & 1 \\
\hline $\mathbf{Z}$ & & -40 & 0 & 0 & 0 & -5 & -5 & 0 & 0 & 1 & 1 & -4 & 1 & 1 & 1 & 1 & 0 & 0 & 5 & 0 & 0 & 0 & 0 \\
\hline
\end{tabular}

De acuerdo con la técnica del método simplex se indica la variable que "sale" de la base es P15 y a su vez la variable que "entra" es P4.

Tabla 5. Resultado de la tercera interacción del método simplex 


\begin{tabular}{cccccccccccccccccccccccccccc}
\hline \multicolumn{2}{c}{ Tabla 3 } & & $\mathbf{0}$ & $\mathbf{0}$ & $\mathbf{0}$ & $\mathbf{0}$ & $\mathbf{0}$ & $\mathbf{0}$ & $\mathbf{0}$ & $\mathbf{0}$ & $\mathbf{0}$ & $\mathbf{0}$ & $\mathbf{0}$ & $\mathbf{0}$ & $\mathbf{0}$ & $\mathbf{0}$ & $\mathbf{- 1}$ & $\mathbf{- 1}$ & $\mathbf{- 1}$ & $\mathbf{- 1}$ & $\mathbf{- 1}$ & $\mathbf{- 1}$ & $\mathbf{- 1}$ \\
\hline Base & Cb & P0 & P1 & P2 & P3 & P4 & P5 & P6 & P7 & P8 & P9 & P10 & P11 & P12 & P13 & P14 & P15 & P16 & P17 & P18 & P19 & P20 & P21 \\
\hline P4 & 0 & 2 & 0 & -1 & -1 & 1 & 1 & 0 & 0 & -1 & 0 & 1 & 0 & 0 & 0 & 0 & 1 & 0 & -1 & 0 & 0 & 0 & 0 \\
\hline P16 & -1 & 1 & 0 & 0 & -1 & 0 & 1 & 0 & 0 & 0 & -1 & 1 & 0 & 0 & 0 & 0 & 0 & 1 & -1 & 0 & 0 & 0 & 0 \\
\hline P1 & 0 & 8 & 1 & 1 & 1 & 0 & 0 & 1 & 1 & 0 & 0 & -1 & 0 & 0 & 0 & 0 & 0 & 0 & 1 & 0 & 0 & 0 & 0 \\
\hline P18 & -1 & 1 & 0 & 1 & 1 & 0 & -1 & -1 & 0 & 1 & 0 & 0 & -1 & 0 & 0 & 0 & -1 & 0 & 0 & 1 & 0 & 0 & 0 \\
\hline P19 & -1 & 3 & 0 & 1 & 1 & 0 & 0 & -1 & -1 & 1 & 0 & 0 & 0 & -1 & 0 & 0 & -1 & 0 & 0 & 0 & 1 & 0 & 0 \\
\hline P20 & -1 & 13 & 0 & 2 & 2 & 0 & 0 & 1 & 0 & 1 & 0 & -1 & 0 & 0 & -1 & 0 & -1 & 0 & 1 & 0 & 0 & 1 & 0 \\
\hline P21 & -1 & 12 & 0 & 1 & 2 & 0 & 0 & 1 & 1 & 1 & 0 & -1 & 0 & 0 & 0 & -1 & -1 & 0 & 1 & 0 & 0 & 0 & 1 \\
\hline $\mathbf{Z}$ & & -30 & 0 & -5 & -5 & 0 & 0 & 0 & 0 & -4 & 1 & 1 & 1 & 1 & 1 & 1 & 5 & 0 & 0 & 0 & 0 & 0 & 0 \\
\hline
\end{tabular}

De acuerdo con la técnica del método simplex se indica la variable que "sale" de la base es P18 y a su vez la variable que "entra" es $\mathrm{P} 2$. 
Tabla 6. Resultado de la cuarta interacción del método simplex

\begin{tabular}{cccccccccccccccccccccccc}
\hline \multicolumn{1}{c}{ Tabla 4 } & & $\mathbf{0}$ & $\mathbf{0}$ & $\mathbf{0}$ & $\mathbf{0}$ & $\mathbf{0}$ & $\mathbf{0}$ & $\mathbf{0}$ & $\mathbf{0}$ & $\mathbf{0}$ & $\mathbf{0}$ & $\mathbf{0}$ & $\mathbf{0}$ & $\mathbf{0}$ & $\mathbf{0}$ & $\mathbf{- 1}$ & $\mathbf{- 1}$ & $\mathbf{- 1}$ & $\mathbf{- 1}$ & $\mathbf{- 1}$ & $\mathbf{- 1}$ & $\mathbf{- 1}$ \\
\hline Base & Cb & P0 & P1 & P2 & P3 & P4 & P5 & P6 & P7 & P8 & P9 & P10 & P11 & P12 & P13 & P14 & P15 & P16 & P17 & P18 & P19 & P20 & P21 \\
\hline P4 & 0 & 3 & 0 & 0 & 0 & 1 & 0 & -1 & 0 & 0 & 0 & 1 & -1 & 0 & 0 & 0 & 0 & 0 & -1 & 1 & 0 & 0 & 0 \\
\hline P16 & -1 & 1 & 0 & 0 & -1 & 0 & 1 & 0 & 0 & 0 & -1 & 1 & 0 & 0 & 0 & 0 & 0 & 1 & -1 & 0 & 0 & 0 & 0 \\
\hline P1 & 0 & 7 & 1 & 0 & 0 & 0 & 1 & 2 & 1 & -1 & 0 & -1 & 1 & 0 & 0 & 0 & 1 & 0 & 1 & -1 & 0 & 0 & 0 \\
\hline P2 & 0 & 1 & 0 & 1 & 1 & 0 & -1 & -1 & 0 & 1 & 0 & 0 & -1 & 0 & 0 & 0 & -1 & 0 & 0 & 1 & 0 & 0 & 0 \\
\hline P19 & -1 & 2 & 0 & 0 & 0 & 0 & 1 & 0 & -1 & 0 & 0 & 0 & 1 & -1 & 0 & 0 & 0 & 0 & 0 & -1 & 1 & 0 & 0 \\
\hline P20 & -1 & 11 & 0 & 0 & 0 & 0 & 2 & 3 & 0 & -1 & 0 & -1 & 2 & 0 & -1 & 0 & 1 & 0 & 1 & -2 & 0 & 1 & 0 \\
\hline P21 & -1 & 11 & 0 & 0 & 1 & 0 & 1 & 2 & 1 & 0 & 0 & -1 & 1 & 0 & 0 & -1 & 0 & 0 & 1 & -1 & 0 & 0 & 1 \\
\hline Z & & -25 & 0 & 0 & 0 & 0 & -5 & -5 & 0 & 1 & 1 & 1 & -4 & 1 & 1 & 1 & 0 & 0 & 0 & 5 & 0 & 0 & 0 \\
\hline
\end{tabular}

Fuente: Elaboración propia, PHP-Simplex

De acuerdo con la técnica del método simplex se indica la variable que "sale" de la base es P16 y a su vez la variable que "entra" es P5.

Tabla 7. Resultado de la cuarta interacción del método simplex 


\begin{tabular}{|c|c|c|c|c|c|c|c|c|c|c|c|c|c|c|c|c|}
\hline \multicolumn{3}{|c|}{ Tabla 1} & -1 & -1 & -1 & -1 & -1 & -1 & -1 & $\mathbf{0}$ & $\mathbf{0}$ & $\mathbf{0}$ & $\mathbf{0}$ & $\mathbf{0}$ & $\mathbf{0}$ & $\mathbf{0}$ \\
\hline Base & $\mathbf{C b}$ & P0 & P1 & $\mathbf{P 2}$ & P3 & P4 & P5 & P6 & P7 & P8 & P9 & P10 & P11 & P12 & P13 & P14 \\
\hline P4 & -1 & 5 & -1 & 0 & 0 & 1 & 0 & 0 & 0 & 0 & 0 & 1 & -1 & 1 & -1 & 0 \\
\hline P5 & -1 & 33 & 0.6 & 0 & 0 & 0 & 1 & 0 & 0 & 0 & -3 & 0 & 0,6 & -1 & 0,6 & $-0,3$ \\
\hline P7 & -1 & 13 & 0.6 & 0 & 0 & 0 & 0 & 0 & 1 & 0 & $-0,3$ & 0 & 0,3 & $1,11 \mathrm{E}-03$ & 0,6 & $-0,3$ \\
\hline $\mathbf{P 2}$ & -1 & 23 & 0.6 & 1 & 0 & 0 & 0 & 0 & 0 & 0 & $-0,3$ & 0 & 0,3 & $-1,00 \mathrm{E}-03$ & 0,3 & 0.6 \\
\hline P3 & -1 & 23 & 0.6 & 0 & 1 & 0 & 0 & 0 & 0 & 0 & 0,6 & -1 & 0,6 & -1 & 0,6 & $-0,3$ \\
\hline P6 & -1 & 2 & -1 & 0 & 0 & 0 & 0 & 1 & 0 & 0 & 0 & 0 & 0 & 1 & -1 & 0 \\
\hline P8 & 0 & 16 & -16 & 0 & 0 & 0 & 0 & 0 & 0 & 1 & $-0,6$ & 1 & $-0,6$ & 1 & $-0,6$ & $-0,6$ \\
\hline $\mathbf{Z}$ & & -16 & 0.3 & 0 & 0 & 0 & 0 & 0 & 0 & 0 & 0,3 & 0 & 0,3 & 0 & 0,3 & 0,3 \\
\hline
\end{tabular}

Hay infinitos valores de X1, X2, X3, X4, X5, X6, X7 para el valor óptimo Z = 16, los cuales están contenidos en la región del espacio $11 \mathrm{X} 1+1 \mathrm{X} 2+1 \mathrm{X} 3+1 \mathrm{X} 4+1 \mathrm{X} 5+1 \mathrm{X} 6+1 \mathrm{X} 7=16$ que cumple las restricciones del problema.

\section{Una de ellas es:}

$$
\begin{aligned}
& X 1=1 \\
& X 2=2 \\
& X 3=2 \\
& X 4=5 \\
& X 5=3 \\
& X 6=2 \\
& X 7=1
\end{aligned}
$$


La solución óptima para este modelo de programación lineal de siete variables y siete restricciones se muestra en el modelo matemático representado en su forma estándar, y pasar por el método de las dos fases, es decir, considera en cada una de las variables que "salen" del modelo y aquellas que "entran", representada en la tabla requerida del método simplex. Las decisiones gerenciales respecto a la asignación de turnos de trabajo, van en función de los presupuestos asignados de la matriz, de modo que las variables de decisión se vuelven más complejas en agencias de mayor tamaño para la contratación de personal de cajas y el tipo de trabajadores de esta modalidad de trabajo se suele programarlos en función de las necesidades de atención, por lo que para este tipo de modelos es importante que para este caso de empleados es necesario indicar la franja horaria aplicada a su turno de trabajo y de esta forma la cuota de trabajadores sea la mínima necesaria.

\section{CONCLUSIONES}

La programación lineal en cualquiera de sus técnicas es útil para el diseño de algoritmos que suplan las necesidades empresariales, ya que ayudan a optimizar las operaciones diarias reduciendo costos que son importantes para las empresas, dado que estos recursos son limitados. Las estrategias de contratación dependen de las decisiones que los gerentes toman a diario, sin embargo, estas decisiones pueden mejorar con el uso de técnicas de investigación y metodologías que se encuentran a disposición de todos. Afortunadamente en la era de la digitalización y automatización de procesos se dispone de software especializado, que ayudan a reducir en cuestión de segundos el tiempo de la generación de cálculos para obtener resultados óptimos y dar respuesta a problemas de asignación y planificación óptima de horarios de trabajo, generando ahorros significativos para apoyar de manera efectiva en la toma de decisiones.

El objetivo de minimizar los recursos y los costos involucrados permite identificar la solución óptima factible, que tiene impactos financieros favorables a las empresas en los márgenes de beneficio dada la creciente complejidad de los negocios y en la gestión de atención al cliente. Este tipo de modelo es complementario a los estudios de teorías de colas y líneas de espera, cuya base de estudio constituye un reto para la mejora continua de las operaciones en las agencias de servicio como son los bancos, cooperativas, mutualistas, supermercados y todas aquellas actividades que involucren la atención a personas en ambientes laborales en donde la calidad de vida depende mucho del uso de la tecnología. En el caso explícito de este estudio, se logró dar una solución básica factible 
al problema de asignación de horarios de la agencia bancaria, tomando en cuenta los horarios de trabajo, validando el modelo de siete restricciones con siete variables, verificando que los turnos cubran las necesidades de la agencia con personal de medio tiempo para trabajar 8h diarias cinco días a la semana y dos días de descanso del personal de cajas en la jornada laboral.

Son pocas las organizaciones que en el Ecuador utilizan para las necesidades reales la programación matemática, apenas el 19,9\% de las organizaciones en este país han incorporado a su quehacer laboral y de actividades la aplicación de esta ciencia y arte, y a la utilización de medios online y offline para lograr mayor difusión de sus productos, servicio o soluciones en el mercado local o internacional; un 28,6\% de empresas no utilizan para nada marketing dentro de sus modelos de negocio (Villarreal, Montenegro, \& Nuñez, 2021), por lo que el reto es apropiado para los profesionales cuyo interés esté en encontrar óptimos para maximizar beneficios o minimizar costos.

\section{REFERENCIAS BIBLIOGRAFICAS}

Acuña, S., Madiedo, E., \& Ortíz, N. (Junio de 2013). Modelo de programación lineal binaria para el balance de carga de trabajo en el problema de asignación de proyectos. Ingeniería y Universidad, 17, 167-181.

Adisbán. (27 de Enero de 2021). escuelacajeros. Recuperado el 26 de Julio de 2021, de https://escuelacajeros.com/cuanto-gana-un-cajero-bancario-en-ecuador/

Arias, J., Bautista, D., \& Meneses, C. (2019). Revisión de literatura sobre los modelos de optimización en programación de turnos de enfermería. UIS Ingenierías, 18(2), 245-258.

Bermúdez, Y. (7 de Julio de 2011). Aplicaciones de programación lineal, entera y mixta. Ingeniería industral, actualidad y nuevas tendencias, 2(7), 85-104.

Cavada, J., Cortés, C., \& Rey, P. (28 de Septiembre de 2012). Modelo de planificación y asignación de personal para el patio de equipajes de un aeropuerto internacional. Claio SBPO, 2033-2045.

Coll, V., \& Olga, B. (2006). Evaluación de la eficiencia mediante análisis envolvente de datos. Valencia: Universidad de Valencia.

De la Fuente, D., Lozano, J., \& Isabel, F. (1998). Asignación de turnos mediante metaehurísticas, aplicación en empresas asturianas. Revista Asturiana de Economía, 12, 275-287. 
Izar, J. (1996). Fundamentos de investigación de operaciones. En Fundamentos de investigación de operaciones para administraciòn (págs. 63-88). San Luis Potosí: Universitaria Potosina.

León, M., Díaz, R., \& Contreras, O. (2007). Utilización del modelo de transporte para la asignación de trabajos a máquinas considerando prioridades. Ingeniería, 11(2), 47-65.

Mariel, O., Aleixo, J., \& Alvares, D. (3 de Mayo de 2019). Modelos matemáticos para la determinación del turno óptimo en plantaciones forestales. Madera y Bosques, 25(1), 1-19.

Mathstools. (5 de Agosto de 2021). mathstools.com. Recuperado el 6 de agosto de 2021, de

https://www.mathstools.com/section/main/Metodo_de_las_Dos_Fases?lang=es\# .YQyj2IhKg2x

Miranda, J. P. (Septiembre de 2017). Programación entera para el diseño de jornadas laborales de reponedores en la industria de retail. Ingeniería de sistemas, XXXI, 65-79.

Ojeda, R. (Junio de 2015). Toma de Decisiones en la Asignación de Personal a Horarios de Trabajo Empleando Programación Lineal: El Caso de una Empresa de Atención Telefónica en México. Investigación administrativa, 44.

Pérez, W., Navarro, N., \& Rocha, J. (22 de Julio de 2019). Modelo de Planeación y Asignación de Personal en la Industria de Servicios con Programación Entera Mixta. Easychair, 1-7.

Render, B., Stair, R., \& Hanna, M. (2012). Métodos cuantitativos para los negocios. Ciudad de México: Pearson.

Soot, T. (2018). Matemáticas Aplicadas a los negocios, las Ciencias Sociales y de la Vida. Toluca: Cengage Learning.

Taha, H. (2011). Investigación de operaciones. México: Pearson.

Taha, H. (2012). Investigación de operaciones. Ciudad de México: Pearson.

Terrazas, R. (5 de Septiembre de 2012). Aplicación de la programación matemática a la localización de proyectos. Perspectivas, 29, 71-94. Obtenido de http://www.scielo.org.bo/pdf/rp/n29/n29a04.pdf 
Villarreal, L., Montenegro, D., \& Nuñez, J. (14 de Abril de 2021). Optimización Matemática como Herramienta para la Toma de Decisiones en la Empresa. Ingenio, III, 40-60. 